\title{
Comparison of Presentation and Clinical Outcome between Children and Young Adults with Differentiated Thyroid Cancer
}

\author{
Jian-Tao Wang ${ }^{1,2 \&}$, Rui Huang ${ }^{1 \&}$, An-Ren Kuang1*
}

\begin{abstract}
Background: The aim of the present study was to evaluate the presentation, clinical course and outcome between children and young adults with differentiated thyroid cancer (DTC) treated in our hospital. Materials and Methods: The medical records of 145 patients with DTC who underwent surgery followed by radioiodine and thyroid hormone (TSH) suppression were retrospectively reviewed. The follow up was between January 2006 and June 2012. These patients consisted of 38 children (age $\leq 18 y$ ) and 107 young adult patients (age $\leq 30 \mathrm{y}$ ). The clinical characteristics and outcome were analyzed and compared, and the progression-free survival (PFS) was evaluated using the Kaplan-Meier method. Results: At initial diagnosis, a greater degree of extra thyroidal extension was found in children than adults patients $(p<0.001)$. However, there was no significant difference between the two groups with regard to the tumor size and the presence of lymph node or distant metastasis $(p=0.172, p=0.050$ and $p=0.068$, respectively). The extent of surgery and the cumulative or mean dose of radioiodine were similar in both groups. During the follow up, the overall survival rate was $100 \%$ for both groups, and the PFS rate was similar in children and in young adults group ( $\log$ rank test, $\left.\chi^{2}=0.126, p=0.723\right)$. Conclusions: In comparison to the young adult patients, DTC in children presents with more aggressive behavior, but outcomes are similar between the two groups after the intensive management of surgery followed by radioiodine and TSH suppression therapy.
\end{abstract}

Keywords: Differentiated thyroid cancer - children - young adults - total thyroidectomy - radioiodine therapy

Asian Pac J Cancer Prev, 15 (17), 7271-7275

\section{Introduction}

Well differentiated thyroid cancer (DTC) in juveniles is a relative rare disease, which accounts for $1.5 \%-3.0 \%$ of all childhood cancers. Recently, the incidence rates of thyroid cancer significantly increased among men and women of all ages all over the world, however, reasons for rising incidence rates were not clearly known yet, as the early diagnosis due to enhanced medical scrutiny and radiation exposure in the childhood may be part of the reasons (Davies et al., 2014).

Compared to the adult patients, the children patients with DTC tend to present more often with advanced disease than adults at the time of diagnosis. However, detailed recommendations for thyroid cancer in the American Thyroid Association (ATA) guidelines primarily addressed approach for treating adult patients without particular notice to the children patients. The treatment strategies for the differentiated thyroid cancer (DTC) in adults include surgery, radioiodine (131I) and the thyroid hormone (TSH) suppression therapy. The combination of the three modalities has been applied at varying levels of intensity, as the optional treatment modality is still controversial (Cooper et al., 2009).
A previous study suggested that DTC was more aggressive in pre-puberty compared to puberty, however, a similar result was shown after a combination of initial intensive treatment including extensive surgery and high dose radioiodine ablation followed by TSH suppression (Lazar et al., 2009). Are the clinical characteristic and the clinical outcome different between the children and young adult patients with DTC? Until now, only few articles have analyzed pediatric thyroid patients verse young adults about the clinical characteristic and the clinical outcome (Kim et al., 2012; Agac et al., 2013). We aimed to evaluate the differences between the children and young adults DTC patients about the clinical presentation, clinical course and clinical outcome in present study.

\section{Materials and Methods}

Between Jan 2006 and Jan 2012, more than eight hundred patients with DTC were treated in the Department of Nuclear Medicine, West China Hospital. 145 of them were under the age of 30 years old. The medical records of these patients were retrospectively reviewed. None of the patients had head and neck irradiation history. Age range at diagnosis was 6-30 years old, including 38 children 
( $\leq 18$ years old) and 107 young adults (19-30 years old). Follow up duration was 6-80 months. All the patients underwent surgery followed by radioiodine treatment and TSH suppression. Three surgeons had been performed the thyroid surgeries of these patients. The surgery procedure included total or near total thyroidectomy in our hospital. Preoperative neck ultrasonography and chest CT scans were used for the assessment of cervical lymph node and pulmonary metastasis. Postoperative data about tumor characteristics included histological type, tumor size, extra-thyroidal extension, number of neoplastic foci, and local and distant metastases. Postoperative data on tumor characteristics included histological type, tumor size, extra-thyroidal extension, number of neoplastic foci, and local and distant metastases. American Joint Committee on Cancer/Union International Concer Control pathological TNM classification criteria was used to define the extrathyroidal extension.

After surgery, all patients were advised to have low iodine diet 3 weeks before the radioiodine therapy which usually began at 4 weeks after withdrawal of Levpthyroxine. Radioiodine therapy was administered using fixed doses empirically, taking into account of the site and extent of metastatic disease: 2.96 to $3.70 \mathrm{GBq}$ (80$100 \mathrm{mCi})$ for thyroid remnant ablation, $5.55 \mathrm{GBq}(150 \mathrm{mCi})$ for disease confined to the neck, and $7.40 \mathrm{GBq}(200 \mathrm{mCi})$ for distant metastasis. All patients underwent a posttherapy whole-body scan (WBS) 5 days after radioiodine administration. Blood samples for determination of serum TSH, thyroglobulin (Tg) and thyroidglobulin anti-body $(\mathrm{TgAb})$ were taken just before radioiodine administration and after the therapy.

Follow-up consisted of detailed clinical examinations, testing of thyroid function and determination of serum $\mathrm{Tg}$ and anti-Tg antibodies. Intervals between examinations ranged from 3 to 6 months, depending on the estimated risk of disease recurrence. Neck ultrasound scan, chest radiograph, diagnostic WBS with 131I (74MBq), and serum $\mathrm{Tg}$ assay (off thyroid hormone therapy) were performed every 6 to 12 months within the first 2 years after diagnosis. In patients appearing to be in complete remission (defined as negative diagnostic results on WBS and undetectable or low serum Tg levels (less than $2 \mathrm{ng} / \mathrm{ml}$ in thyroid hormone off state), both tested with thyrotropin stimulation, accompanied by the absence of anatomically definable disease on neck ultrasound scanning or chest computed tomography), repeated evaluation was performed every 2 years for 4 years and every 5 years thereafter.

Repeated 131I treatments were given to patients with evidence of local recurrence, persistent disease or distant metastasis. The definition of recurrence was the reappearance of disease after complete remission. There were no deaths own to the favorable prognosis. ProgressFree Survival (PFS) was used as the end point of the analysis to assess the outcome. Progressive disease was considered when new foci of radioiodine uptake were found on the WBS or new tumor lesion was anatomically definable on the neck ultrasound or chest computer tomography. PFS was defined as the time from the combination treatment of operation and thyroid remnant ablation until the progress event, if not occurred, until the last follow-up visit to our hospital.

TNM stage adopted in this article followed American Joint Committee on cancer classification (2009) and the risk stratification for recurrence used for the post-operate patients was based on the ATA guidelines for the thyroid cancer.

This retrospective study was approved by the institutional review board of West China Hospital.

\section{Statistical analysis}

SPSS for windows version 18.0 (SPSS, Chicago, Illinois) was used for the data analysis. Differences in the two groups were tested with independent-samples $\mathrm{t}$ tests for continuous variables presented as mean (SD) and $\chi^{2}$ tests or Fisher exact tests for categorical variables. PFS was evaluated with the Kaplan-Meier method. Comparison of the overall PFS in children and young adult patients was examined with the log-rank test. Statistical significance was defined as $p<0.05$.

\section{Results}

Patient characteristic and DTC clinical presentation Of the 145 patients younger than 30 years old diagnosed as DTC, 38 were children (less than 18 years old) and 107 were young adults (19-30 years old). The mean age at the diagnosis was significantly different between the 2 groups $((14.8 \pm 3.8) v s .(26.1 \pm 2.8), p<0.001$; Table 1). Among these patients, 132 patients $(91.0 \%)$ had been diagnosed with papillary thyroid carcinoma and 13 $(9.0 \%)$ had been diagnosed with follicular carcinoma; the constitution of the pathology types was not statistically different between the 2 groups $(p=0.744)$. At diagnosis, extra-thyroidal extension of the tumor was more prevalent in children patients than that of young adult patients ( $p<0.001$; Table 1). Although there was no statistically significant difference, the mean tumor size was larger in children than that in the young adults $((2.83 \pm 1.23) \mathrm{cm}$ vs. $(2.50 \pm 1.30) \mathrm{cm}, p=0.172$; Table 1$))$, and the positive nodal disease was more frequent in the children than that of the young adults $(81.6 \%$ (31/38) vs. $60.7 \%(75 / 107)$, $p=0.050$; Table 1$)$. There was no statistically significant difference in the frequency of intra-thyroidal multifocality and distant metastasis between the two groups. 15 patients had distant metastases, and all of them were lung metastasis. The risk stratification of the patients according to the ATA guideline was significantly different which suggested that the children patients were more likely to suffer recurrence $(p<0.001$; Table 1$)$.

\section{Initial treatment}

All patients in both groups underwent the same initial treatment protocol, consisting of total thyroidectomy with dissection of suspicious enlarged lymph nodes and radioiodine therapy (Table 2). All patients underwent total thyroidectomy or near total thyroidectomy, and the type of operation was similar in the two groups ( $p=0.900$; Table 2). There were no significant differences in the frequency of central and lateral neck dissection between the two groups ( $p=0.675$ and $p=0.866$, respectively; Table 2$)$. 
Cumulative and mean radioiodine doses were summarized in Table 2 . Although there was no statistically significant difference, the mean administered dose was higher in the children than that in the young adult patients ((311.6 \pm 285.0$) \mathrm{mCi} v s$. $(234.8 \pm 224.2) \mathrm{mCi}, p=0.094$; Table 2).

\section{Clinical course and outcome}

The complications due to the surgical and radioiodine treatment were comparable between the two groups (Table 3 ). Recurrent laryngeal nerve damage was observed in 1 (2.6\%) patient in the children group and $2(2.8 \%)$ in the young adults group $(p=0.956$; Table 3$)$. The development of hypoparathyroidism (transient or permanent) was documented in $7(18.4 \%)$ children patients and 23 $(21.5 \%)$ in young adult patients $(p=0.688$; Table 3$)$. Short

Table 1. Comparison of the Clinical Presentation between the Children and Adult Patients with Differentiated Thyroid Cancer

\begin{tabular}{|c|c|c|c|}
\hline & Children & Young adults & $P$-value \\
\hline Gender (F/M) & $38(31 / 7)$ & $107(79 / 28)$ & 0.385 \\
\hline Age(mean/SD/range) & $\begin{array}{c}14.8 / 3.8 / \\
6-18\end{array}$ & $\begin{array}{c}26.1 / 2.8 / \\
19-30\end{array}$ & $<0.001$ \\
\hline Tumor size(mean/SD/range) & $\begin{array}{c}2.83 / 1.23 / \\
0.5-6.0\end{array}$ & $\begin{array}{c}2.50 / 1.30 / \\
0.2-7.0\end{array}$ & 0.172 \\
\hline Extrothyroidal extension, n (\%) & $28(73.7)$ & $40(37.4)$ & $<0.001$ \\
\hline Histology type, n (\%) & & & 0.744 \\
\hline $\mathrm{P}$ & $34(89.5)$ & $98(91.6)$ & \\
\hline $\mathrm{F}$ & $4(10.5)$ & $9(8.4)$ & \\
\hline T stage, $\mathrm{n}(\%)$ & & & $<0.001$ \\
\hline 1 & $4(10.5)$ & $43(40.2)$ & \\
\hline 2 & $3 \quad(7.9)$ & $25(23.4)$ & \\
\hline 3 & 17 (44.7) & $27(25.2)$ & \\
\hline 4 & $14(36.9)$ & $12(11.2)$ & \\
\hline $\mathrm{N}$ stage, $\mathrm{n}(\%)$ & & & 0.05 \\
\hline 0 & 7 (18.4) & $42(39.3)$ & \\
\hline 1a & 12 (31.6) & $30(28.0)$ & \\
\hline $1 b$ & $19(50.0)$ & $35(32.7)$ & \\
\hline M stage, n (\%) & & & 0.068 \\
\hline 0 & 31 (81.6) & $99(92.5)$ & \\
\hline 1 & $7(18.4)$ & $8(7.5)$ & \\
\hline Multifocal disease, n (\%) & & & 0.122 \\
\hline Unifocal & $25(65.8)$ & $85(79.4)$ & \\
\hline Multifocal & $13(34.2)$ & $22(20.6)$ & \\
\hline Risk stratification, n (\%) & & & $<0.001$ \\
\hline Low & $5(13.2)$ & $38(35.5)$ & \\
\hline Intermediate & $14(36.8)$ & $51(47.7)$ & \\
\hline High & $19(50)$ & $18(16.8)$ & \\
\hline
\end{tabular}

Table 2. Comparison of Treatment Management between the Children and Adult Patients with Differentiated Thyroid Cancer

\begin{tabular}{|c|c|c|c|}
\hline & Children & Young adults & $P$-value \\
\hline \multicolumn{3}{|l|}{ Surgery procedure } & 0.9 \\
\hline Total thyroidectomy, n (\%) & $23(60.5)$ & $66(61.7)$ & \\
\hline Near total thyroidectomy, $\mathrm{n}(\%)$ & $15(39.5)$ & $41(38.3)$ & \\
\hline \multicolumn{4}{|l|}{ Neck dissection, $\mathrm{n}(\%)$} \\
\hline Central dissection & $28(73.7)$ & $75(70.1)$ & 0.675 \\
\hline Lateral dissection & $13(34.2)$ & $35(32.7)$ & 0.866 \\
\hline \multicolumn{3}{|c|}{ Postoperative Radioiodine treatment $(\mathrm{mCi}), \mathrm{n}(\%)$} & 0.06 \\
\hline$\leq 200$ & $21(55.3)$ & $76(71.0)$ & \\
\hline $201-500$ & $10(26.3)$ & $20(18.7)$ & \\
\hline $501-999$ & $4(10.5)$ & $8(7.5)$ & \\
\hline$\geq 1000$ & $3(7.9)$ & $3(2.8)$ & \\
\hline \multicolumn{4}{|l|}{ Radioiodine dose, mean(SD), mCi } \\
\hline & $\begin{array}{c}311.6 \\
(285.0)\end{array}$ & $\begin{array}{c}234.8 \\
(224.2)\end{array}$ & 0.094 \\
\hline
\end{tabular}

Table 3. Comparison of the Side Events and Outcome between the Children Aand Adult Patients with Differentiated Thyroid Cancer

Children Young adults P-value

Post operative complications

Recurrent laryngeal nerve damage $1(2.6) \quad 3(2.8) \quad 0.956$

hypoparathyroidism

total

transient

$7(18.4) \quad 23(21.5) \quad 0.688$

Permanent

$\begin{array}{lll}5(13.2) & 19(17.8) & 0.512\end{array}$

Postoperative radioiodine therapy

Short term adverse event

Nausea and vomiting

Neck pain

Sialadenitis

Long term adverse event

Mild myelosuppression

Leukemia

Secondary malignancy

Recurrence patients

2(5.2) $\quad 4(3.7) \quad 0.686$

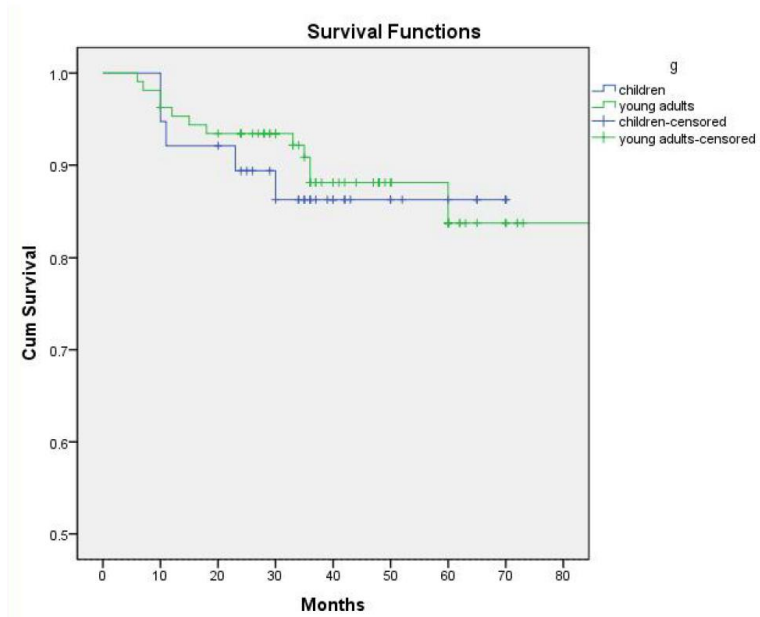

Figure 1.Progress Free Survival between the Children And Adult Patients With Differentiated Thyroid Cancer. g, group

term complications after the radioiodine therapy such as gastritis, neck pain, and sialadenitis were not rare in both groups, although these adverse effects were well tolerated and temporary. Mild myelosuppression was observed in 1 child and in 2 young adults ( $p=0.777$; Table 2 ). The mean follow up time was similar in the 2 groups (35 months (12-65 months) in children vs. 38 months (10-70 months), $p=0.561$; Table 2). At the end of the follow up no one suffered the second malignant after the initial treatment. During the follow up, the recurrence in the thyroid bed or lymph nodes occurred in 17 patients, of them 5 (13.2\%) were children patients and $12(11.2 \%)$ were young adult patients ( $p=0.750$; Table 2). The Kaplan-Meier ProgressFree Survival (PFS) curves showed that PFS rate was similar in the children and the young adults group (logrank test, $\left.\chi^{2}=0.126, \mathrm{df}=1, p=0.723\right)$. The overall survival rate in both groups was $100 \%$ (Figure 1).

None of the 15 patients ( 7 children and 8 young adults) with pulmonary metastasis achieved completely response: 6 of the 7 children patients and 7 of the 8 young adult patients with lung metastasis were radioiodine uptake; the rest of them were radioiodine resistant. Until the last follow-up time, most of the metastatic patients 
demonstrated decreased serum Tg or extent of metastatic lesion in the imaging modalities compared to the initial evaluation.

\section{Discussion}

DTC has long been considered to constitute a distinct disease in children and in adults (Miller et al., 1995). However, only a few studies have showed the differences in clinical characteristics between children and young adult patients with DTC (Kim et al., 2012; Agac et al., 2013).

This retrospective study found that tumor size was larger and extra-thyroidal extension was more prevalent in children than that in the young adults patients at initial diagnosis, which are consistent with previous reports. It should be considered that the thyroid gland is smaller in children than in young adults, which could lead to earlier involvement of the thyroid capsule and surrounding tissues (Farah et al., 1999). DTC in children patients present more aggressive behavior compared to that in young adults according to the TNM classification, which summarizes the local regional and distant metastasis. However, it was of little use as all children/young adults have stage I/II disease.

Multifocal seems to be more frequent in the children patients, however, there are no statistically significant. Frankenthaler and Danese reported that $90 \%$ children with thyroid cancer will have PTC, typically presenting with a lump in the thyroid or the lateral neck, without other symptoms (Frankenthaler et al., 1990; Danses et al., 1997). As the previous report, we found that, in this study, $89.5 \%$ children patients were PTC, compared to that of $91.5 \%$ in the young adult patients.

The treatment of the children thyroid cancer remains controversial. Resection is the key for DTC children patients just as the adult patients, however, the surgery extent is still being debated (Yu et al., 2013). For most of the children patients with multifocal or regional lymph node involvement, routine near total or total thyroidectomy were recommended by some investigators who argued that such surgery can remove all malignant thyroid tissue, decrease recurrence risk and improve patient outcome(Handkiewicz et al., 2007). On the contrary, others advocated less aggressive treatment to decrease the risk of surgical complications (Gulcelik et al., 2012). Prospective clinical trials are good means to end the debate, however, the low incidence and excellent prognosis of DTC have hampered its performing.

Radioiodine ablation was demonstrated to be an independent factor which could reduce local recurrence, distant metastasis, and cancer death (Degroot et al., 1990). Additionally, post operation radioiodine remnant ablation and TSH suppression therapy make follow up more reliable: improving subsequent WBS and Tg sensitivity, and allowing earlier diagnosis and greater effectiveness of repeated radioiodine therapy for patients with persistent or metastatic disease. Uptake of the ${ }^{131}$ I depends on the sodium iodide symporter $(N I S)$ gene expression, the level of which is different between the children and adults patients, although reduced compared with that of healthy thyroid cells, the former have greater and more frequently detectable expression, implying greater differentiation and radioiodine responsiveness, which may be relevant to the outcome (Ringel et al., 2001; Patel et al., 2002; Faggino et al., 2004).

TSH suppression in adults and children with DTC improves survival rate and prolongs recurrence time, especially in the children, because DTC cells have been found to be very sensitive to TSH in this age group. The lack of stimulation manifested in undetectable TSH levels could serve to act against tumor cell proliferation and thus reduce the risk of recurrence (Hovens et al., 2007).

Long-term follow-up shows better disease specific survival in children than in adults despite of more advanced presentation in children with DTC. Survival over decades is common, even with distant metastasis. Genetics studies of thyroid cancer found that RET mutations and $R E T / P T C$ rearrangements were most detected in the childhood patients, while RRAF or RAS gene mutation in the adults patients (Lima et al., 2004). Interestingly, all $R E T / P T C$-related subtypes show more regional metastasis but result in more differentiated pathological type with better prognosis (Adeniran et al., 2006; Mehta et al., 2012). It may be associated with NIS gene expression, which improves the 131I uptake so as to advocate radioiodine therapy in the DTC children patients. Higher level of NIS gene expression was usually found in the children patients with $R E T / P T C$ mutations, while lower level in the adult patients with $R R A F$ gene mutation (Riesco et al., 2006; Lupi et al., 2007).

Recently, in a review, Zaman pointed out that an adequately performed total or near thyroidectomy ensures use of either low or no adjuvant radioiodine therapy in the low risk DTC patients and can minimize the probabilities of second primary malignancy and adverse effects (Zaman et al., 2013). Radioiodine is also not recommended by some thyroid societies in the low risk patients for lacking significant impact and increased side effects, of which a major concern is that the radioiodine therapy may potentially be a risk factor for second primary malignancy. In the present study, extensive surgery including total/near total throidectomy with or without lymph node dissection followed radioiodine therapy and TSH suppression was taken for both the children and young adult patients, and most of the patients, including low risk patients, showed excellent prognosis without severe adverse events after the surgery and radioiodine treatment during the follow up. However, follow-up durations in present study were not long enough to detect the long-term adverse events. Well designed studies with larger sample size and longtime follow-up were needed to confirm our findings in the near future.

In the current study, children patients with DTC seemed to be more likely to recur, on account of the significantly higher rate of the high risk patients compared to that in the young adults according to the ATA risk stratification. However, during the follow up, the recurrence rate was similar in both groups. The reasons may be as follows: first, the definition of recurrence was reappearance of the tumor lesion after complete remission, but children patients with distant metastasis classified as high risk was 
not cured at the end of the follow up; second, the children patients may favor more from the radical combination of extensive surgery and followed radioiodine and TSH suppression to reduce the recurrence rate; third, the insufficient follow-up durations may partially account for the phenomenon.

In conclusion, Our present data showed that children patients with DTC have more aggressive behavior compared to the young adult patients. Despite of the advanced disease at initial diagnosis, the children patients have similar prognosis as the young adults subject to the intensive management, which suggested that the more intensive initial management may be essential to avoid recurrence in young patients with DTC.

\section{Acknowledgements}

This work was supported by National Natural Science Foundation of China (81471692; 81071184).

\section{References}

Adeniran A, Zhu Z, Gandhi M, et al (2006). Correlation between genetic alterations and microscopic features, clinical manifestations, and prognostic characteristics of thyroid papillary carcinomas. Am J Surg Pathol, 30, 216-22.

Agac A, Kutun S, Çetin A (2013). Are the characteristics of thyroid cancer different in young patients? J Pediatr Endocrinol Metab, 27,497-502.

Cooper D, Doherty G, Haugen B, et al (2009). Revised american thyroid association management guidelines for patients with thyroid nodules and differentiated thyroid cancer: the american thyroid association (ATA) guidelines taskforce on thyroid nodules and differentiated thyroid cancer. Thyroid, 19, 1167-214.

Davies L, Welch H (2014). Current Thyroid Cancer Trends in the United States. JAMA Otolaryngol Head Neck Surg, 140, 317-22.

Danese D, Gardini A, Farsetti A, et al (1997). Thyroid carcinoma in children and adolescents. Eur J Pediatr, 156, 190-4.

Degroot L, Kaplan E, McCormick M, et al (1990). Natural history, treatment, and course of papillary thyroid carcinoma. $J$ Clin Endocrinol Metab, 71, 414-24.

Faggiano A, Coulot J, Bellon N, et al (2004). Age-dependent variation of follicular size and expression of iodine transporters in human thyroid tissue. J Nucl Med, 45, 232-7.

Farahati J, Reiners C, Demidchik E (1999). Is the UICC/ AJCC classification of primary tumor in childhood thyroid carcinoma valid? J Nucl Med, 40, 2125.

Frankenthaler R, Sellin R, Cangir A, et al (1990). Lymph node metastasis from papillary-follicular thyroid carcinoma in young patients. Am J Surg, 160, 341-3.

Gulcelik M, Kuru B, Dincer H, et al (2012). Complications of completion versus total thyroidectomy. Asian Pac J Cancer Prev, 13, 5225-8.

Handkiewicz-Junak D, Wloch J, Roskosz J, et al (2007). Total thyroidectomy and adjuvant radioiodine treatment independently decrease locoregional recurrence risk in childhood and adolescent differentiated thyroid cancer. $J$ Nucl Med, 48, 879-88.

Hovens G, Stokkel M, Kievit J, et al (2007). Associations of serum thyrotropin concentrations with recurrence and death in differentiated thyroid cancer. J Clin Endocrinol Metab, 92, 2610-5.
Kim SS, Kim SJ, Kim IJ, et al (2012). Comparison of clinical outcomes in differentiated thyroid carcinoma between children and young adult patients. Clin Nucl Med, 37, 850-3.

Lazar L, Lebenthal Y, Steinmetz A, et al (2009). Differentiated thyroid carcinoma in pediatric patients: comparison of presentation and course between pre-pubertal children and adolescents. J Pediatr, 154, 708-14.

Lima J, Trovisco V, Soares P, et al (2004). BRAF mutations are not a major event in post-Chernobyl childhood thyroid carcinomas. J Clin Endocrinol Metab, 89, 4267-71.

Lupi C, Giannini R, Ugolini C, et al (2007). Association of BRAF V600E mutation with poor clinicopathological outcomes in 500 consecutive cases of papillary thyroid carcinoma. J Clin Endocrinol Metab, 92, 4085-90.

Mehta V, Nikiforov Y, Ferris R (2013). Use of molecular biomarkers in FNA specimens to personalize treatment for thyroid surgery. Head Neck. 35, 1499-506.

Miller R, L Young J, Novakovic B (1995). Childhood cancer. Cancer, 75, 395-405.

Patel A, Jhiang S, Dogra S, et al (2002). Differentiated thyroid carcinoma that express sodium-iodide symporter have a lower risk of recurrence for children and adolescents. Pediatr Res, 52, 737-44.

Riesco G, Gutierrez P, Garcia M, et al (2006). The oncogene BRAFV600E is associated with a high risk of recurrence and less differentiated papillary thyroid carcinoma due to the impairment of $\mathrm{Na}+/ \mathrm{I}-$ targeting to the membrane. Endocr relat cancer, 13, 257-69.

Ringel M, Anderson J, Souza S, et al (2001). Expression of the sodium iodide symporter and thyroglobulin genes are reduced in papillary thyroid cancer. Mod Pathol, 14, 289-96.

Yu WB, Tao SY, Zhang NS (2012). Is level V dissection necessary for low-risk patients with papillary thyroid cancer metastasis in lateral neck levels II, III, and IV. Asian Pac J Cancer Prev, 13, 4619-22.

Zaman MU, Fatima N, Padhy AK, et al (2013).Controversies about radioactive iodine-131 remnant ablation in low risk thyroid cancers: are we near a consensus? Asian Pac J Cancer Prev, 14, 6209-13. 